\title{
Universality and $m_{X}$ cut effects in $B \rightarrow X_{s} \ell^{+} \ell^{-}$
}

\author{
Keith S.M. Lee, ${ }^{1}$ Zoltan Ligeti, ${ }^{2,1}$ Iain W. Stewart, ${ }^{1}$ and Frank J. Tackmann ${ }^{2}$ \\ ${ }^{1}$ Center for Theoretical Physics, Massachusetts Institute of Technology, Cambridge, MA 02139 \\ ${ }^{2}$ Ernest Orlando Lawrence Berkeley National Laboratory, University of California, Berkeley, CA 94720
}

\begin{abstract}
The most precise comparison between theory and experiment for the $B \rightarrow X_{s} \ell^{+} \ell^{-}$rate is in the low $q^{2}$ region, but the hadronic uncertainties associated with an experimentally required cut on $m_{X}$ potentially spoil the search for new physics in these decays. We show that a $10-30 \%$ reduction of $\mathrm{d} \Gamma\left(B \rightarrow X_{s} \ell^{+} \ell^{-}\right) / \mathrm{d} q^{2}$ due to the $m_{X}$ cut can be accurately computed using the $B \rightarrow X_{s} \gamma$ shape function. The effect is universal for all short distance contributions in the limit $m_{X}^{2} \ll m_{B}^{2}$, and this universality is spoiled neither by realistic values of the $m_{X}$ cut nor by $\alpha_{s}$ corrections. Both the differential decay rate and forward-backward asymmetry with an $m_{X}$ cut are computed.
\end{abstract}

\section{INTRODUCTION}

In the standard model (SM) the flavor-changing neutral current process $B \rightarrow X_{s} \ell^{+} \ell^{-}$does not occur at tree level and is thus a sensitive probe of new physics. Predicting its rate involves integrating out the $W, Z$, and $t$ at a scale of order $m_{W}$ by matching on to the Hamiltonian [1], 2]

$$
H_{W}=-\frac{G_{F}}{\sqrt{2}} V_{t b} V_{t s}^{*}\left[\sum_{i=1}^{6} C_{i} O_{i}+\frac{1}{4 \pi^{2}} \sum_{i=7}^{10} C_{i} O_{i}\right],
$$

evolving to $\mu=m_{b}$, and computing matrix elements of $H_{W}$. Here $O_{1}-O_{6}$ are four-quark operators and

$$
\begin{aligned}
O_{7} & =\bar{m}_{b} \bar{s} \sigma_{\mu \nu} e F^{\mu \nu} P_{R} b, \\
O_{8} & =\bar{m}_{b} \bar{s} \sigma_{\mu \nu} g G^{\mu \nu} P_{R} b, \\
O_{9} & =e^{2}\left(\bar{s} \gamma_{\mu} P_{L} b\right)\left(\bar{\ell} \gamma^{\mu} \ell\right), \\
O_{10} & =e^{2}\left(\bar{s} \gamma_{\mu} P_{L} b\right)\left(\bar{\ell} \gamma^{\mu} \gamma_{5} \ell\right),
\end{aligned}
$$

where $P_{L, R}=\left(1 \mp \gamma_{5}\right) / 2$. Measurements of $C_{7,8,9,10}$ probe flavor-changing neutral currents and test the SM. This can be done with the dilepton invariant mass spectrum, $d \Gamma / d q^{2}$, with $q^{2}=\left(p_{\ell^{+}}+p_{\ell^{-}}\right)^{2}$. It is calculable in an operator product expansion (OPE), and the nonperturbative corrections are $\mathcal{O}\left(\Lambda_{\mathrm{QCD}}^{2} / m_{b}^{2}\right)$ [3, 迆. The matching and anomalous dimensions for $C_{i}$ are known at next-tonext-to-leading log (NNLL) order, as are the perturbative QCD corrections to the matrix elements of $O_{i}$ [5, 6, 7] (except small $\mathrm{O}_{3-6}$ terms).

A complication in $B \rightarrow X_{s} \ell^{+} \ell^{-}$compared with $B \rightarrow$ $X_{s} \gamma$ is that the long distance contributions, $B \rightarrow J / \psi X_{s}$ and $\psi^{\prime} X_{s}$ followed by $J / \psi, \psi^{\prime} \rightarrow \ell^{+} \ell^{-}$, are two orders of magnitude above the short distance prediction, a fact which is not well understood. Therefore, either theory and data are both interpolated, or the short distance calculation is compared with the data for $q^{2}<m_{J / \psi}^{2}$ or $q^{2}>m_{\psi^{\prime}}^{2}$. The low $q^{2}$ region, $q^{2}<6 \mathrm{GeV}^{2}$, allows the most precise comparison with the SM, but requires a cut on the invariant mass of the hadronic final state, $m_{X}<m_{X}^{\text {cut }}$. In the latest Belle analysis $m_{X}^{\text {cut }}=2 \mathrm{GeV}$ 8], while Babar uses $m_{X}^{\text {cut }}=1.8 \mathrm{GeV}$ [9]. This cut is to remove backgrounds and will likely be required for quite some time [10]. So far, its effect has been studied only in the Fermi-motion model [11]. [The high $q^{2}$ region is unaffected by the $m_{X}$ cut, but the rate is lower, and calculating it involves an expansion in $\Lambda_{\mathrm{QCD}} /\left(m_{b}-\sqrt{q^{2}}\right)$.]

In this letter we compute the $B \rightarrow X_{s} \ell^{+} \ell^{-}$rate with an $m_{X}$ cut in the low $q^{2}$ region in a model-independent framework. For $\left(m_{X}^{\text {cut }}\right)^{2}=\mathcal{O}\left(\Lambda_{\mathrm{QCD}} m_{b}\right)$, the local OPE used in all earlier analyses breaks down and must be replaced by an OPE involving $b$ quark distribution functions (shape functions), as explained below. We will compute

$$
\Gamma_{i j}^{\text {cut }}=\int_{q_{1}^{2}}^{q_{2}^{2}} \mathrm{~d} q^{2} \int_{0}^{m_{X}^{\text {cut }}} \mathrm{d} m_{X} \operatorname{Re}\left(c_{i} c_{j}^{*}\right) \frac{\mathrm{d}^{2} \Gamma_{i j}}{\mathrm{~d} q^{2} \mathrm{~d} m_{X}},
$$

and study the ratios

$$
\eta_{i j}\left(m_{X}^{\text {cut }}, q_{1}^{2}, q_{2}^{2}\right)=\frac{\Gamma_{i j}^{\text {cut }}}{\Gamma_{i j}^{0}} .
$$

For convenience we define normalization factors

$$
\begin{aligned}
\Gamma_{i j}^{0} & =\frac{\Gamma_{0}}{m_{B}^{5}} \int_{q_{1}^{2}}^{q_{2}^{2}} \mathrm{~d} q^{2} \operatorname{Re}\left(c_{i} c_{j}^{*}\right) \frac{\left(m_{b}^{2}-q^{2}\right)^{2}}{m_{b}^{3}} G_{i j}, \\
\Gamma_{0} & =\frac{G_{F}^{2} m_{B}^{5}}{192 \pi^{3}} \frac{\alpha_{\mathrm{em}}^{2}}{4 \pi^{2}}\left|V_{t b} V_{t s}^{*}\right|^{2},
\end{aligned}
$$

with kinematic dependence $G_{99}=G_{00}=\left(2 q^{2}+m_{b}^{2}\right)$, $G_{77}=4 m_{B}^{2}\left(1+2 m_{b}^{2} / q^{2}\right)$, and $G_{79}=12 m_{B} m_{b}$. Here and below, $m_{b}$ is a short distance mass, such as $m_{b}^{1 S}$ [12. In Eqs. (3 5), $i j=\{77,99,00,79\}$ label contributions of time-ordered products of operators, $T\left\{O_{j}^{\dagger}, O_{i}\right\}$. The total decay rate with cuts is the sum of these contributions,

$$
\Gamma^{\text {cut }}=\left.\Gamma\right|_{\substack{m_{X}<m_{X}^{\text {cut }} \\ q_{1}^{2}<q^{2}<q_{2}^{2}}}=\sum_{i j} \Gamma_{i j}^{\text {cut }} .
$$

We will also study $\eta_{i j}^{\prime}=\eta_{i j}^{\prime}\left(p_{X}^{+ \text {cut }}, q_{1}^{2}, q_{2}^{2}\right)$, which differ from $\eta_{i j}$ by the replacement of $m_{X}$ by $p_{X}^{+}=E_{X}-\left|\vec{p}_{X}\right|$ :

$$
\eta_{i j}^{\prime}=\frac{1}{\Gamma_{i j}^{0}} \int_{q_{1}^{2}}^{q_{2}^{2}} \mathrm{~d} q^{2} \int_{0}^{p_{X}^{+ \text {cut }}} \mathrm{d} p_{X}^{+} \operatorname{Re}\left(c_{i} c_{j}^{*}\right) \frac{\mathrm{d}^{2} \Gamma_{i j}}{\mathrm{~d} q^{2} \mathrm{~d} p_{X}^{+}} .
$$

In Eqs. (3-7) the short distance coefficients $c_{7,9,0}$ track the $C_{7,9,10}$ dependence in Eq. (1) that one would like to 
measure. Here $c_{7}=C_{7}^{\text {mix }}\left(q^{2}\right), c_{9}=C_{9}^{\text {mix }}\left(q^{2}\right)$, and $c_{0}=$ $C_{10}$ can be obtained from local OPE calculations [13] at each order, as discussed in Ref. [14.

The $\eta_{i j}$ 's contain the effects of the $m_{X}$ cut, and are defined with a normalization that makes them less sensitive to the choice of $i j$. At leading order in $\Lambda_{\mathrm{QCD}} / m_{b}$ and $\alpha_{s}, \eta_{i j}$ give the fraction of events with $m_{X}<m_{X}^{\text {cut }}$, and $\eta_{i j}=1$ for $m_{X}^{\text {cut }}=m_{B}$. This interpretation is altered at subleading order by $\alpha_{s}$ corrections, but knowing $\eta_{i j}$ at a given order in perturbation theory is still sufficient to determine $\Gamma_{i j}^{\text {cut }}$ and thus the total rate with cuts in Eq. (6), at this order. In principle, $\eta_{i j}$ depend in a nontrivial way on $i j$ (and $q_{1}^{2}$ and $q_{2}^{2}$ ) due to different dependence on kinematic variables, $\alpha_{s}$ corrections, etc. At leading order in $\Lambda_{\mathrm{QCD}} / m_{b}$, we demonstrate that $\eta_{i j}$ are actually independent of the choice of $i j$, a property which we call "universality". We first show this formally in section II at leading order in $p_{X}^{+} / m_{B} \ll 1$ for $\eta^{\prime}\left(p_{X}^{+ \text {cut }}\right)$. Then in section III we demonstrate it numerically for the experimentally relevant $\eta\left(m_{X}^{\text {cut }}\right)$, including the $\alpha_{s}$ corrections and phase space effects.

We also compute the decay rate for current experimental cuts. We find that the rate is sensitive to the choice of the cut, and that the cut causes a reduction in the rate by $10-30 \%$. Since the same shape function occurs in $B \rightarrow X_{s} \ell^{+} \ell^{-}, X_{u} \ell \bar{\nu}$, and $X_{s} \gamma$, the $m_{X}^{\text {cut }}$ or $p_{X}^{+ \text {cut }}$ dependence in one can be accurately determined from the others, and the magnitude of the reduction can be computed quite accurately. Alternatively, instead of using the theoretical computation of the $m_{X}^{\text {cut }}$ dependence, universality can be exploited to remove the main uncertainties, by normalizing the $B \rightarrow X_{s} \ell^{+} \ell^{-}$rate to $B \rightarrow X_{u} \ell \bar{\nu}$.

\section{II. $m_{X}$ CUT EFFECTS AT LEADING ORDER}

For simplicity, consider the kinematics in the $B$ meson's rest frame. Since $q=p_{B}-p_{X}$,

$$
2 m_{B} E_{X}=m_{B}^{2}+m_{X}^{2}-q^{2} .
$$

If $m_{X}^{2} \ll m_{B}^{2}$ and $q^{2}$ is not near $m_{B}^{2}$, then $E_{X}=\mathcal{O}\left(m_{B}\right)$. Since $E_{X}^{2} \gg m_{X}^{2}, p_{X}$ is near the light-cone, with $p_{X}^{+}=$ $E_{X}-\left|\vec{p}_{X}\right|=\mathcal{O}\left(\Lambda_{\mathrm{QCD}}\right)$ and $p_{X}^{-}=E_{X}+\left|\vec{p}_{X}\right|=\mathcal{O}\left(m_{B}\right)$. Of the variables symmetric in $p_{\ell^{+}}$and $p_{\ell^{-}}\left(p_{X}^{ \pm}, E_{X}, q^{2}\right.$, $m_{X}^{2}$ ), only two are independent, and we work with $q^{2}$ and $p_{X}^{+}$or $m_{X}$. The phase space cuts are shown in Fig. 1.

For the $p_{X}^{+} \ll p_{X}^{-}$region, factorization of the form $\mathrm{d} \Gamma=H J \otimes \hat{f}^{(0)}$ has been proven for semileptonic and radiative $B$ decays 15], where $H$ contains perturbative physics at $\mu_{b} \sim m_{b}, J$ at $\mu_{i} \sim \sqrt{\Lambda_{\mathrm{QCD}} m_{b}}$, and $\hat{f}^{(0)}(\omega)$ is a universal nonperturbative shape function [16]. This factorization also applies for $B \rightarrow X_{s} \ell^{+} \ell^{-}$with the same $\hat{f}^{(0)}$, as long as $q^{2}$ is not parametrically small 14.

In the $q^{2}<6 \mathrm{GeV}^{2}$ region, $\left|C_{9}^{\operatorname{mix}}\left(q^{2}, \mu_{0}=4.8 \mathrm{GeV}\right)\right|=$ 4.52 to better than $1 \%$, and can be taken to be constant.

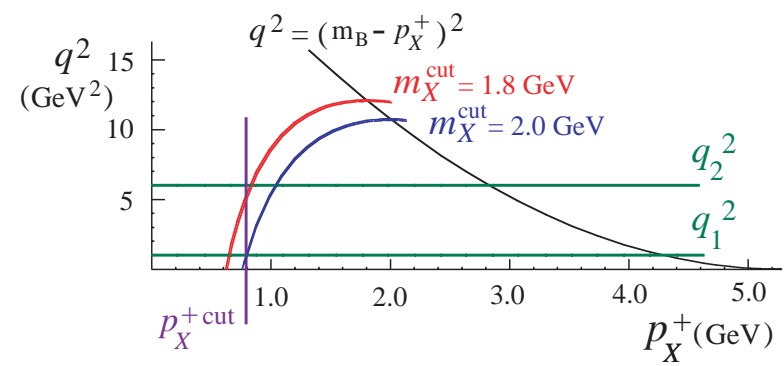

FIG. 1: Phase space cuts. A substantial part of the rate for $q_{1}^{2}<q^{2}<q_{2}^{2}$ falls in the rectangle bounded by $p_{X}^{+}<p_{X}^{+ \text {cut }}$.

We neglect $\alpha_{s}$ corrections in this section and find

$$
\begin{aligned}
\frac{\mathrm{d} \Gamma}{\mathrm{d} p_{X}^{+} \mathrm{d} q^{2}}= & \hat{f}^{(0)}\left(p_{X}^{+}\right) \frac{\Gamma_{0}}{m_{B}^{5}} \frac{\left[\left(m_{B}-p_{X}^{+}\right)^{2}-q^{2}\right]^{2}}{\left(m_{B}-p_{X}^{+}\right)^{3}} \\
\times & \left\{\left(\left|C_{9}^{\text {mix }}\right|^{2}+C_{10}^{2}\right)\left[2 q^{2}+\left(m_{B}-p_{X}^{+}\right)^{2}\right]\right. \\
& +4 m_{B}^{2}\left|C_{7}^{\text {mix }}\right|^{2}\left[1+\frac{2\left(m_{B}-p_{X}^{+}\right)^{2}}{q^{2}}\right] \\
& \left.+12 m_{B} \operatorname{Re}\left[C_{7}^{\text {mix }} C_{9}^{\text {mix* }}\right]\left(m_{B}-p_{X}^{+}\right)\right\},
\end{aligned}
$$

where $\hat{f}^{(0)}(\omega)$ has support in $\omega \in[0, \infty)$. As a function of $p_{X}^{+}$, the kinematic terms in Eq. (9) vary only on a scale $m_{B}$, while $\hat{f}^{(0)}\left(p_{X}^{+}\right)$varies on a scale $\Lambda_{\mathrm{QCD}}$. Writing $m_{B}=m_{b}+\bar{\Lambda}$ and expanding in $\left(p_{X}^{+}-\bar{\Lambda}\right) / m_{B}$ decouple the $p_{X}^{+}$and $q^{2}$ dependences in Eq. (9), and give exactly the local OPE prefactors, $\left(m_{b}^{2}-q^{2}\right)^{2} G_{i j}\left(q^{2}\right)$, used in Eq. (5). For $\eta_{i j}^{\prime}\left(p_{X}^{+ \text {cut }}, q_{1}^{2}, q_{2}^{2}\right)$, the $p_{X}^{+}$integration is over a rectangle in Fig. 1, whose boundaries do not couple $p_{X}^{+}$and $q^{2}$. Thus, with the above expansion, we find $\eta_{i j}^{\prime}=\eta^{\prime}$, where

$$
\eta^{\prime}=\int \mathrm{d} p_{X}^{+} \hat{f}^{(0)}\left(p_{X}^{+}\right)
$$

independent of $i j$ and $q_{1}^{2}, q_{2}^{2}$. While the $m_{X}$ cut retains more events than the $p_{X}^{+}$cut, the latter may give theoretically cleaner constraints on short distance physics when statistical errors become small.

The effect of the $m_{X}$ cut is $q^{2}$ dependent, because the upper limit of the $p_{X}^{+}$integration is $q^{2}$ dependent, as shown in Fig. 11. When we include the full $p_{X}^{+}$dependence in Eq. (9), the universality of $\eta_{i j}\left(m_{X}^{\text {cut }}, q_{1}^{2}, q_{2}^{2}\right)$ is maintained to better than $3 \%$ for $1 \mathrm{GeV}^{2} \leq q_{1}^{2} \leq 2 \mathrm{GeV}^{2}$, $5 \mathrm{GeV}^{2} \leq q_{2}^{2} \leq 7 \mathrm{GeV}^{2}$, and $m_{X}^{\text {cut }} \geq 1.7 \mathrm{GeV}$, because the region where the $p_{X}^{+}$and $q^{2}$ integration limits are coupled has a small effect on the $i j$ dependence. This is exhibited in Fig. 2, where the solid curves show $\eta_{i j}\left(m_{X}^{\text {cut }}, 1 \mathrm{GeV}^{2}, 6 \mathrm{GeV}^{2}\right)$ with the shape function set to model 1 of [17], with $m_{b}^{1 S}=4.68 \mathrm{GeV}$ and $\lambda_{1}$ from [18]. (Taking $q_{1}^{2}=1 \mathrm{GeV}^{2}$ instead of $4 m_{\ell}^{2}$ increases the sensitivity to $C_{9,10}$, but one may be concerned by local duality / resonances near $q^{2}=1 \mathrm{GeV}^{2}$. To estimate this uncertainty, assume the $\phi$ is just below the cut and $\mathcal{B}(B \rightarrow$ 


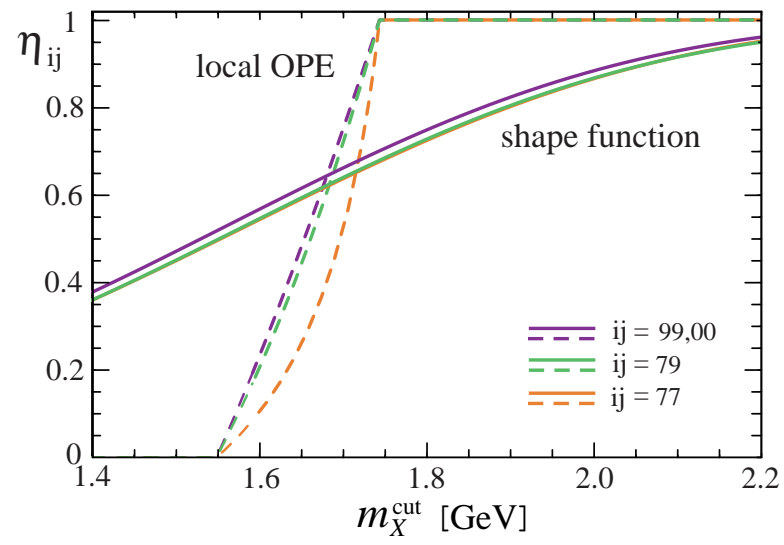

FIG. 2: $\eta_{i j}\left(m_{X}^{\text {cut }}, 1 \mathrm{GeV}^{2}, 6 \mathrm{GeV}^{2}\right)$ as functions of $m_{X}^{\text {cut }}$. The dashed curves show the local OPE result, the solid curves include the leading shape function effects. The uppermost, middle, and lowest curves are $\eta_{00,99}, \eta_{79}$, and $\eta_{77}$, respectively.

$\left.X_{s} \phi\right) \approx 10 \times \mathcal{B}\left(B \rightarrow K^{(*)} \phi\right)$. Then $B \rightarrow X_{s} \phi \rightarrow X_{s} \ell^{+} \ell^{-}$ is $\sim 2 \%$ of the $X_{s} \ell^{+} \ell^{-}$rate.)

The local OPE results for $\eta_{i j}\left(m_{X}^{\text {cut }}, q_{1}^{2}, q_{2}^{2}\right)$ are obtained by replacing $\hat{f}^{(0)}\left(p_{X}^{+}\right)$by $\delta\left(\bar{\Lambda}-p_{X}^{+}\right)$in Eq. (9)). Performing the $p_{X}^{+}$integral sets $\left(m_{B}-p_{X}^{+}\right)=m_{b}$ and implies $m_{X}^{2}>$ $\bar{\Lambda}\left(m_{B}-q^{2} / m_{b}\right)$. This makes the lower limit on $q^{2}$ equal $\max \left\{q_{1}^{2}, m_{b}\left[m_{B}-\left(m_{X}^{\text {cut }}\right)^{2} / \bar{\Lambda}\right]\right\}$, and so the $\eta_{i j}$ 's depend on the shape of $\mathrm{d} \Gamma_{i j}$. In Fig. 2 the local OPE results are shown by dashed lines, and clearly $\eta_{77} \neq \eta_{99}$. However, the local OPE is not applicable for $p_{X}^{+} \sim \Lambda_{\mathrm{QCD}}$.

The universality of $\eta_{i j}$ found here could be broken by $\alpha_{s}$ corrections in the $H$ or $J$ functions, or by renormalization group evolution, since these effects couple $p_{X}^{+}$and $q^{2}$ and have been neglected so far. We consider these next.

\section{CALCULATION AND RESULTS AT $\mathcal{O}\left(\alpha_{s}\right)$}

A complication in calculating $B \rightarrow X_{s} \ell^{+} \ell^{-}$compared with $B \rightarrow X_{u} \ell \bar{\nu}$ is that, in the evolution of the effective
Hamiltonian down to $m_{b}, C_{9}(\mu)$ receives a $\ln \left(m_{W}^{2} / m_{b}^{2}\right)$ enhanced contribution from the mixing of $\mathrm{O}_{2}$. Thus, formally, $C_{9} \sim \mathcal{O}\left(1 / \alpha_{s}\right)$, and conventionally one expands the amplitude in $\alpha_{s}$, treating $\alpha_{s} \ln \left(m_{W}^{2} / m_{b}^{2}\right)=\mathcal{O}(1)$ [13. In the local OPE this is reasonable, since the nonperturbative corrections are small, and at next-to-leading log (NLL) all dominant terms in the rate are included. However, in the shape function region nonperturbative effects are $\mathcal{O}(1)$ and only the rate is calculable, not the amplitude. With the traditional counting, the $C_{9}^{2}$ contribution to the rate would be needed to $\mathcal{O}\left(\alpha_{s}^{2}\right)$ before the $C_{10}^{2}$ terms could be included.

This would be a bad way to organize the perturbative corrections (numerically $\left|C_{9}\left(m_{b}\right)\right| \approx\left|C_{10}\right|$ ). It can be circumvented by using a "split matching" procedure to decouple the perturbation series above and below the scale $m_{b}$ [14]. This allows us to consider the short distance coefficients $C_{7}^{\text {mix }}, C_{9}^{\text {mix }}$, and $C_{10}$ as $\mathcal{O}(1)$ numbers when organizing the perturbation theory at $m_{b}^{2}$ and $m_{b} \Lambda_{\mathrm{QCD}}$.

The rate and the forward-backward asymmetry are

$$
\begin{aligned}
\frac{\mathrm{d}^{2} \Gamma}{\mathrm{d} q^{2} \mathrm{~d} p_{X}^{+}} & =\frac{\Gamma_{0}}{m_{B}^{2}} H\left(q^{2}, p_{X}^{+}\right) F^{(0)}\left(p_{X}^{+}, p^{-}\right), \\
\frac{\mathrm{d}^{2} A_{\mathrm{FB}}}{\mathrm{d} q^{2} \mathrm{~d} p_{X}^{+}} & =\frac{\Gamma_{0}}{m_{B}^{2}} K\left(q^{2}, p_{X}^{+}\right) F^{(0)}\left(p_{X}^{+}, p^{-}\right),
\end{aligned}
$$

where $p^{-}=m_{b}-q^{2} /\left(m_{B}-p_{X}^{+}\right)$. The hard functions $H$ and $K$ were computed in 14] using soft-collinear effective theory (SCET) [19, 20 and split matching. This factorizes the dependence on scales above and below $m_{b}$ as $\Gamma_{i j} \sim H_{1}\left(\mu_{0}\right) H_{2}\left(\mu_{b}\right) F^{(0)}\left(\mu_{b}\right)$, with separate $\mu_{0}$ and $\mu_{b}$ independence. Up to the order one is working at, $H_{1}$ is $\mu_{0}$ independent, the $\mu_{b}$ dependence in $H_{2}$ and $F^{(0)}$ cancels, and $F^{(0)}$ is $\mu_{i}$ independent. The shape function model is specified at $\mu_{\Lambda}$. The convolution of jet and shape functions at NLL including $\alpha_{s}$ corrections is

$$
\begin{aligned}
F^{(0)}\left(p_{X}^{+}, p^{-}\right)= & U_{H}\left(p^{-}, \mu_{i}, \mu_{b}\right)\left\{\hat{f}^{(0)}\left(p_{X}^{+}, \mu_{i}\right)+\frac{\alpha_{s}\left(\mu_{i}\right) C_{F}}{4 \pi}\left[\left(2 \ln ^{2} \frac{p_{X}^{+} p^{-}}{\mu_{i}^{2}}-3 \ln \frac{p_{X}^{+} p^{-}}{\mu_{i}^{2}}+7-\pi^{2}\right) \hat{f}^{(0)}\left(p_{X}^{+}, \mu_{i}\right)\right.\right. \\
& \left.\left.+\int_{0}^{1} \frac{\mathrm{d} z}{z}\left(4 \ln \frac{z p_{X}^{+} p^{-}}{\mu_{i}^{2}}-3\right)\left(\hat{f}^{(0)}\left(p_{X}^{+}(1-z), \mu_{i}\right)-\hat{f}^{(0)}\left(p_{X}^{+}, \mu_{i}\right)\right)\right]\right\}, \\
\hat{f}^{(0)}\left(\omega, \mu_{i}\right)= & \frac{e^{V_{S}\left(\mu_{i}, \mu_{\Lambda}\right)}}{\Gamma(1+\eta)}\left(\frac{\omega}{\mu_{\Lambda}}\right)^{\eta} \int_{0}^{1} \mathrm{~d} t \hat{f}^{(0)}\left[\omega\left(1-t^{1 / \eta}\right), \mu_{\Lambda}\right],
\end{aligned}
$$

where $\eta=(16 / 25) \ln \left[\alpha_{s}\left(\mu_{\Lambda}\right) / \alpha_{s}\left(\mu_{i}\right)\right], U_{H}$ was computed in Ref. [19], the one-loop jet function in Ref. [21, 22], and the shape function evolution up to $\mu_{i}$ in Refs. [19, 22] (for earlier calculations, see Refs. [15, 23]). The hard coefficients 
$H$ and $K$ for $B \rightarrow X_{s} \ell^{+} \ell^{-}$are

$$
\begin{aligned}
H\left(q^{2}, p_{X}^{+}\right)= & \frac{\left[\left(1-\hat{p}_{X}^{+}\right)^{2}-\hat{q}^{2}\right]^{2}}{\left(1-\hat{p}_{X}^{+}\right)^{3}}\left\{\left[\left|C_{9}^{\text {mix }}\left(s, \mu_{0}\right)\right|^{2}+C_{10}^{2}\right]\left[2 \hat{q}^{2} \Omega_{A}^{2}\left(s, \mu_{b}\right)+\left(1-\hat{p}_{X}^{+}\right)^{2} \Omega_{B}^{2}\left(s, \hat{p}_{X}^{+}, \mu_{b}\right)\right]\right. \\
& \left.+4\left|C_{7}^{\text {mix }}\left(\mu_{0}\right)\right|^{2}\left[\Omega_{C}^{2}\left(s, \mu_{b}\right)+\frac{2\left(1-\hat{p}_{X}^{+}\right)^{2}}{\hat{q}^{2}} \Omega_{D}^{2}\left(s, \mu_{b}\right)\right]+12 \operatorname{Re}\left[C_{7}^{\text {mix }}\left(\mu_{0}\right) C_{9}^{\text {mix }}\left(s, \mu_{0}\right)^{*}\right]\left(1-\hat{p}_{X}^{+}\right) \Omega_{E}\left(s, \mu_{b}\right)\right\} \\
K\left(q^{2}, p_{X}^{+}\right)= & -\frac{3 \hat{q}^{2}\left[\left(1-\hat{p}_{X}^{+}\right)^{2}-\hat{q}^{2}\right]^{2}}{\left(1-\hat{p}_{X}^{+}\right)^{3}} \Omega_{A}\left(s, \mu_{b}\right) \operatorname{Re}\left\{C_{10}^{*}\left[C_{9}^{\text {mix }}\left(s, \mu_{0}\right) \Omega_{A}\left(s, \mu_{b}\right)+\frac{2\left(1-\hat{p}_{X}^{+}\right)}{\hat{q}^{2}} C_{7}^{\text {mix }}\left(\mu_{0}\right) \Omega_{D}\left(s, \mu_{b}\right)\right]\right\},(13)
\end{aligned}
$$

where $s=q^{2} / m_{b}^{2}, \hat{q}^{2}=q^{2} / m_{B}^{2}, \hat{p}_{X}^{+}=p_{X}^{+} / m_{B}$, and

$$
\begin{array}{ll}
\Omega_{A}=1+\frac{\alpha_{s}}{\pi} \omega_{a}^{V}\left(s, \mu_{b}\right), & \Omega_{B}=1+\frac{\alpha_{s}}{\pi}\left[\omega_{a}^{V}\left(s, \mu_{b}\right)+\frac{\left(1-\hat{p}_{X}^{+}\right)^{2}-\hat{q}^{2}}{2\left(1-\hat{p}_{X}^{+}\right)^{2}} \omega_{b}^{V}(s)+\omega_{c}^{V}(s)\right], \\
\Omega_{C}=1+\frac{\alpha_{s}}{\pi} \omega_{a}^{T}\left(s, \mu_{b}\right), & \Omega_{D}=1+\frac{\alpha_{s}}{\pi}\left[\omega_{a}^{T}\left(s, \mu_{b}\right)-\omega_{c}^{T}(s)\right], \quad \Omega_{E}=\frac{1}{3}\left(2 \Omega_{A} \Omega_{D}+\Omega_{B} \Omega_{C}\right) .
\end{array}
$$

Here $\alpha_{s}=\alpha_{s}\left(\mu_{b}\right)$ and $\omega_{i}^{V, T}$ are defined in Ref. 14.

For each shape function model, the deviations of the $\eta_{i j}$ 's from being universal, with all NLL corrections, are still below $3 \%$. Thus, the picture of universality in Fig. 2 remains valid at NLL order. For this reason we can explore the overall shift by just studying $\eta_{00}$.

In Fig. 3 we plot $\eta_{00}\left(m_{X}^{\text {cut }}, 1 \mathrm{GeV}^{2}, 6 \mathrm{GeV}^{2}\right)$, including the $\alpha_{s}$ corrections. We use ten different models for $\hat{f}^{(0)}$. Our base model has five parameters, three of which are chosen to obey the known constraints on its moments [22], converted to the $1 S$ mass scheme used here. For each of five different choices of the remaining two parameters, we choose two values of the scale, $\mu_{\Lambda}$, where the model is specified. The choice of these ten models is guided by making them consistent with the $B \rightarrow X_{s} \gamma$ data. The ten orange, green and purple (medium, light, dark) curves correspond to $m_{b}^{1 S}=4.68 \mathrm{GeV}, 4.63 \mathrm{GeV}$, and $4.73 \mathrm{GeV}$, respectively, with the central values $\mu_{0}=$ $\mu_{b}=4.8 \mathrm{GeV}$ and $\mu_{i}=2.5 \mathrm{GeV}$. The curves with slightly

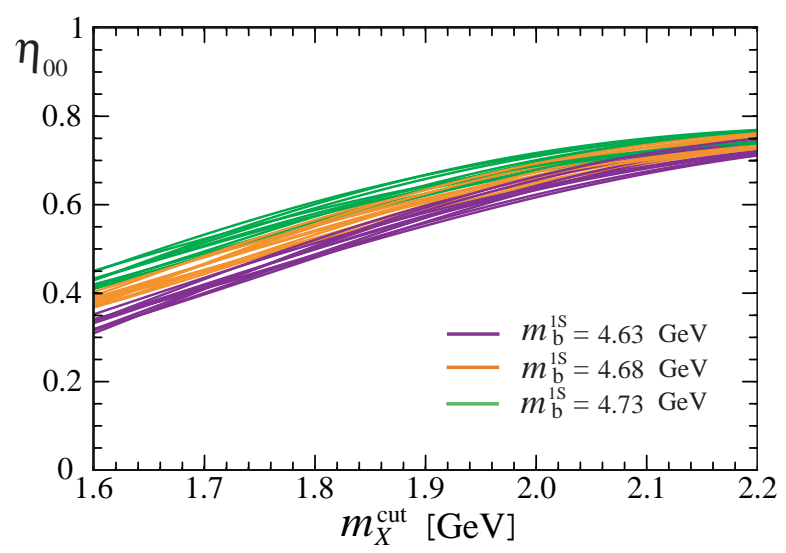

FIG. 3: $\eta_{00}\left(m_{X}^{\text {cut }}, 1 \mathrm{GeV}^{2}, 6 \mathrm{GeV}^{2}\right)$ as a function of $m_{X}^{\text {cut }}$. The orange, green and purple (medium, light, dark) curves show $m_{b}^{1 S}=4.68 \mathrm{GeV}, 4.63 \mathrm{GeV}$, and $4.73 \mathrm{GeV}$, respectively. lower [higher] values of $\eta_{00}$ at large $m_{X}^{\text {cut }}$ correspond to $\mu_{\Lambda}=1.5 \mathrm{GeV}[2 \mathrm{GeV}]$. The spread in the curves gives our determination of the uncertainty from the choice of shape function model and from $m_{b}$. For $m_{X}^{\text {cut }}=2 \mathrm{GeV}$, varying $\mu_{b}$ in the range $3.5 \mathrm{GeV}<\mu_{b}<7.5 \mathrm{GeV}$ changes $\eta_{00}$ by $\pm 6 \%$. We find a $\pm 5 \%$ variation for $2 \mathrm{GeV}<\mu_{i}<3 \mathrm{GeV}$.

Using the $c_{i}$ 's at NLL, for $1 \mathrm{GeV}^{2}<q^{2}<6 \mathrm{GeV}^{2}$ we obtain cut branching ratios

$$
\Gamma^{\text {cut }} \tau_{B}= \begin{cases}(1.20 \pm 0.15) \times 10^{-6} & {\left[m_{X}^{\text {cut }}=1.8 \mathrm{GeV}\right]} \\ (1.48 \pm 0.14) \times 10^{-6} & {\left[m_{X}^{\text {cut }}=2.0 \mathrm{GeV}\right]}\end{cases}
$$

where uncertainties are included from $m_{b}, \mu_{b}, \mu_{i}$, and $\hat{f}^{(0)}$. Changing $\mu_{0}$ to $3.5 \mathrm{GeV}(10 \mathrm{GeV})$ changes both of these rates by $-2 \%(+7 \%)$, and this uncertainty will be reduced by including NNLL corrections [5, 6, 7].

The largest source of universality breaking in the $\eta_{i j}$ 's and one of the largest uncertainties in the cut rate is due to subleading shape functions, which affect the rate by $\sim 5 \%$ for $m_{X}^{\text {cut }}=2 \mathrm{GeV}$ and by $\sim 10 \%$ for $m_{X}^{\text {cut }}=$ $1.8 \mathrm{GeV} 24$.

If the $m_{X}^{\text {cut }}$ dependence were not universal, it would modify the zero of the forward-backward asymmetry, $A_{\mathrm{FB}}\left(q_{0}^{2}\right)=0$. For $m_{X}^{\text {cut }}=2 \mathrm{GeV}$ we find at NLL $\Delta q_{0}^{2} \approx-0.04 \mathrm{GeV}^{2}$, much below the higher order uncertainties [6, 7]. However, we obtain $q_{0}^{2}=2.8 \mathrm{GeV}^{2}$, lower than earlier results [6]. The reason is that in the SCET calculation of $A_{\mathrm{FB}}$, using $K$ in Eq. (13), the pole mass $m_{b}^{\text {pole }}$ never occurs, only $m_{B}-p_{X}^{+}$and $\bar{m}_{b}$ (at this order, $C_{7}^{\text {mix }}=\left(\bar{m}_{b} / m_{B}\right) C_{7}^{\text {eff }}$ [14]). Thus, schematically, $q_{0}^{2} \sim 2 m_{b}\left[\bar{m}_{b}\left(\mu_{0}\right) C_{7}^{\text {eff }}\left(\mu_{0}\right)\right] / \operatorname{Re}\left[C_{9}^{\text {eff }}\left(q_{0}^{2}\right)\right]$, and there is no reason to expand $\bar{m}_{b}$ in terms of $m_{b}^{\text {pole }}$.

In the above analysis, the nonperturbative shape function $f^{(0)}$ was extracted from moments and the $B \rightarrow X_{s} \gamma$ energy spectrum, and this was used as input in determining our $B \rightarrow X_{s} \ell^{+} \ell^{-}$results. The overall $10 \%$ theoretical uncertainty in this approach could be reduced by raising the $m_{X}^{\text {cut }}$. An alternative approach would be to 
keep $m_{X}^{\text {cut }}<m_{D}$ and measure

$$
R=\frac{\Gamma^{\text {cut }}\left(B \rightarrow X_{s} \ell^{+} \ell^{-}\right)}{\Gamma^{\text {cut }}\left(B \rightarrow X_{u} \ell \bar{\nu}\right)},
$$

with the same cuts used in the numerator and denominator. The dependence of the semileptonic rate on $m_{X}^{\text {cut }}$ is identical to that of $\Gamma_{00}^{\text {cut }}$. A measurement of $R$ bypasses the need for a shape function model, because we found that the $m_{X}$-cut effects are universal to a very good approximation and therefore cancel between the numerator and denominator of $R$.

In conclusion, we pointed out that the observed $B \rightarrow$ $X_{s} \ell^{+} \ell^{-}$rate in the low $q^{2}$ region is sensitive to the experimental upper cut on $m_{X}$. The reduction in the rate due to this cut is determined by the universal $B$ shape function. In the region of the experimental measurements an OPE exists only for the decay rate and not for the amplitude, a fact that necessitates a reorganization of the usual perturbation expansion. Since one can use the shape function measured in other processes, the sensitivity to new physics is not reduced. We found that the $\eta$ 's for the different operators' contributions are universal to a good approximation. These results also apply for $B \rightarrow X_{d} \ell^{+} \ell^{-}$, which may be studied at a higher luminosity $B$ factory. Subleading $\Lambda_{\mathrm{QCD}} / m_{b}$ as well as NNLL corrections to the rate and the forward-backward asymmetry will be studied in a separate publication [24.

Acknowledgments We thank Bjorn Lange for helpful discussions. This work was supported in part by the Director, Office of Science, and Offices of High Energy and Nuclear Physics of the U.S. Department of Energy under the Contract DE-AC02-05CH11231 (Z.L. and F.T.), and the cooperative research agreement DOEFC02-94ER40818 (K.L. and I.S.). I.S. was also supported in part by the DOE OJI program and by the Sloan Foundation.
[1] B. Grinstein et al., Nucl. Phys. B 319, 271 (1989).

[2] G. Buchalla, A. J. Buras and M. E. Lautenbacher, Rev. Mod. Phys. 68, 1125 (1996) [hep-ph/9512380].

[3] A. F. Falk, M. E. Luke and M. J. Savage, Phys. Rev. D 49, 3367 (1994) [hep-ph/9308288].

[4] A. Ali et al., Phys. Rev. D 55, 4105 (1997) [hep$\mathrm{ph} / 9609449$.

[5] C. Bobeth, M. Misiak and J. Urban, Nucl. Phys. B 574 (2000) 291 [hep-ph/9910220]; H. H. Asatryan et al., Phys. Rev. D 65 (2002) 074004 [hep-ph/0109140]; C. Bobeth et al., JHEP 0404 (2004) 071 [hep-ph/0312090].

[6] A. Ghinculov et al., Nucl. Phys. B 648 (2003) 254 [hepph/0208088]; H. M. Asatrian et al., Phys. Rev. D 66 (2002) 094013 [hep-ph/0209006].

[7] A. Ghinculov et al., Nucl. Phys. B 685 (2004) 351 [hep$\mathrm{ph} / 0312128]$.

[8] M. Iwasaki et al. [Belle Collaboration], hep-ex/0503044.

[9] B. Aubert et al. [BABAR Collaboration], Phys. Rev. Lett. 93, 081802 (2004) [hep-ex/0404006].

[10] J. Berryhill, SLAC-INT Workshop, Seattle, http://www. int.washington. edu/talks/WorkShops/int_05_1/.

[11] A. Ali and G. Hiller, Phys. Rev. D 60 (1999) 034017 [hep-ph/9807418].

[12] A. H. Hoang, Z. Ligeti and A. V. Manohar, Phys. Rev. D 59, 074017 (1999) [hep-ph/9811239]; Phys. Rev. Lett. 82 (1999) 277 [hep-ph/9809423].

[13] A. J. Buras and M. Munz, Phys. Rev. D 52, 186 (1995) [hep-ph/9501281]; M. Misiak, Nucl. Phys. B 393 (1993) 23 [Erratum-ibid. B 439 (1995) 461].

[14] K. S. M. Lee and I. W. Stewart, hep-ph/0511334.

[15] G. P. Korchemsky and G. Sterman, Phys. Lett. B 340 (1994) 96 [hep-ph/9407344].

[16] M. Neubert, Phys. Rev. D 49 (1994) 3392 [hepph/9311325]; ibid. 4623 [hep-ph/9312311]; I. I. Y. Bigi et al., Int. J. Mod. Phys. A 9 (1994) 2467 [hep-ph/9312359].

[17] F. J. Tackmann, Phys. Rev. D 72 (2005) 034036 [hep$\mathrm{ph} / 0503095]$.

[18] C. W. Bauer et al., Phys. Rev. D 70 (2004) 094017 [hep$\mathrm{ph} / 0408002]$.

[19] C. W. Bauer, S. Fleming and M. E. Luke, Phys. Rev. D 63, 014006 (2001) [hep-ph/0005275]; C. W. Bauer et al., Phys. Rev. D 63, 114020 (2001) [hep-ph/0011336].

[20] C. W. Bauer and I. W. Stewart, Phys. Lett. B 516, 134 (2001) [hep-ph/0107001]; C. W. Bauer, D. Pirjol and I. W. Stewart, Phys. Rev. D 65, 054022 (2002) [hep$\mathrm{ph} / 0109045]$.

[21] C. W. Bauer and A. V. Manohar, Phys. Rev. D70, 034024 (2004) [hep-ph/0312109].

[22] S. W. Bosch et al., Nucl. Phys. B699, 335 (2004) [hep$\mathrm{ph} / 0402094]$.

[23] A. K. Leibovich, I. Low and I. Z. Rothstein, Phys. Rev. D 62 (2000) 014010 [hep-ph/0001028];

[24] K. S. M. Lee et al., to appear. 